hep-th/0601072

\title{
Handbook on string decay
}

\author{
Roberto Iengo $^{a}$ and Jorge G. Russo ${ }^{b}$ \\ ${ }^{a}$ International School for Advanced Studies (SISSA) \\ Via Beirut 2-4, I-34013 Trieste, Italy \\ INFN, Sezione di Trieste \\ ${ }^{b}$ Institució Catalana de Recerca i Estudis Avançats (ICREA), \\ Departament ECM, Facultat de Física, Universitat de Barcelona, Spain
}

\begin{abstract}
We explain simple semi-classical rules to estimate the lifetime of any given highlyexcited quantum state of the string spectrum in flat spacetime. We discuss both the decays by splitting into two massive states and by massless emission. As an application, we study a solution describing a rotating and pulsating ellipse which becomes folded at an instant of time - the "squashing ellipse". This string interpolates between the folded string with maximum angular momentum and the pulsating circular string. We explicitly compute the quantum decay rate for the corresponding quantum state, and verify the basic rules that we propose. Finally, we give a more general (4-parameter) family of closed string solutions representing rotating and pulsating elliptical strings.
\end{abstract}




\section{Decay rate due to breaking}

In general, a string can decay either by emitting light particles or by splitting into two massive strings. Which channel is dominant depends on the string state and on the number of uncompact dimensions. In the situation of splitting into two massive strings with masses much greater than $l_{s}^{-1}$, the two outgoing strings are highly excited string states which admit a classical description. The decay rate in this case can be estimated by semiclassical arguments. In this section we will discuss the different situations in detail and give an estimate of the decay rate due to breaking in each case.

\subsection{Open strings}

The probability of breaking for an open string was studied in [1]. There it was suggested that it is constant along the string and proportional to $g_{o}^{2}$. We propose that, more precisely, the probability per unit time of breaking on a given point and at a given instant is

$$
P_{o}=\frac{g_{o}^{2}}{T}
$$

where $T$ is the period of oscillations of the string (so the probability of breaking in one period is $T P_{o}=g_{o}^{2}$ in agreement with [1]).

In the gauge $X_{0}=\frac{T}{\pi} \tau$, we have

$$
M=\frac{1}{2 \pi \alpha^{\prime}} \int_{0}^{\pi} d \sigma \dot{X}_{0}=\frac{T}{2 \pi \alpha^{\prime}},
$$

or $T=2 \pi \alpha^{\prime} M$. This formula applies to both open and closed strings. The decay rate for an open string is thus obtained by multiplying $P_{o}$ by the number of points of the string $L / l_{s}$, where $l_{s}=\sqrt{\alpha^{\prime}}$ and $L$ is the length of the string, and by the number of "instants" in one period $T / l_{s}$. We find

$$
\Gamma_{\text {open }} \cong\left(\frac{L}{l_{s}}\right)\left(\frac{T}{l_{s}}\right) P_{o} \cong g_{o}^{2} \frac{L}{l_{s}^{2}}
$$

in agreement with [1]. This formula can be checked against the explicit quantum calculation of the decay rate for the open string with maximum angular momentum performed in [2]. It was found that $\Gamma_{\text {open }} \cong g_{o}^{2} M$, where $M=L / l_{s}^{2}$ for this particular string state, in precise agreement with (1.2). We have reproduced this quantum calculation independently. Figure 1a shows $\Gamma_{\text {open }}(M)$ obtained from the exact evaluation of $\operatorname{Im}\left(\Delta M^{2}\right)$ at one loop.

\subsection{Closed strings}

In closed string theory, in the absence of D-branes, a closed string can only break into two outgoing closed strings. This means that the breaking is possible only if two points of the string meet. The possible configurations are: 
- The closed string is folded, with two sides in permanent contact.

- The closed string becomes folded only at some instant of time.

- The closed string has two points which are in permanent contact.

- The closed string has two points which get in contact only at some instant of time.

- The distance between any two points of the string is $\gg l_{s}$ at all times. In this case the string is "unbreakable", meaning that the decay rate into two massive $\left(M_{1,2} \gg l_{s}^{-1}\right)$ string states is exponentially suppressed, $\Gamma=O\left(e^{-c M^{2}}\right)$.

We will assume that the probability of breaking at a given point once in a period is

given by $P_{o}=\frac{g_{o}^{2}}{T}$, as proposed above. This is a natural assumption since the breaking is a local phenomenon which should not depend on global properties of the string, i.e. whether the string is closed or open. In addition, for the breaking to take place, it is necessary that the string simultaneously breaks at two points which are in contact. Here 'simultaneously' means within an interval of order $l_{s}$.

In what follows we apply these simple rules and estimate the decay rate for all the cases listed above.

\subsubsection{Folded string}

The two folds of the closed string are in permanent contact, so the string can break at any time. We can estimate the decay rate by viewing it as two open strings on the top of each other. The breaking can take place only if at a given time the two open strings break at the same point, up to an uncertainty of order $l_{s}$.

The decay rate is thus

$$
\Gamma_{\text {folded }} \cong\left(\frac{L}{l_{s}}\right)\left(\frac{T}{l_{s}}\right) P_{o} P_{o} l_{s} \cong g_{s}^{2} \frac{L}{l_{s} T} \cong g_{s}^{2} \frac{L}{l_{s}^{3} M}, \quad g_{s}=g_{o}^{2} .
$$

In particular, for the folded string with maximum angular momentum $\mathrm{J}_{\max }$, one has $L \cong l_{s}^{2} M$, so that

$$
\Gamma_{\mathrm{J}_{\max }} \cong g_{s}^{2} l_{s}^{-1}
$$

Remarkably, it is constant independent of $M$. This estimate applies at large $N=$ $\alpha^{\prime} M^{2} / 4$. This can be compared with the exact quantum calculation of the decay rate. This was computed in [3], and here we have added more numerical data, up to $N=119$. Figure $1 \mathrm{~b}$ shows the decay rate as a function of the mass. We see that it asymptotically approaches to a constant, confirming the semiclassical estimate.

The decay rates of figure $1 \mathrm{a}$ and $1 \mathrm{~b}$ contain also the channels where one of the emitted particles is massless. The semiclassical estimate, however, assumes that both 

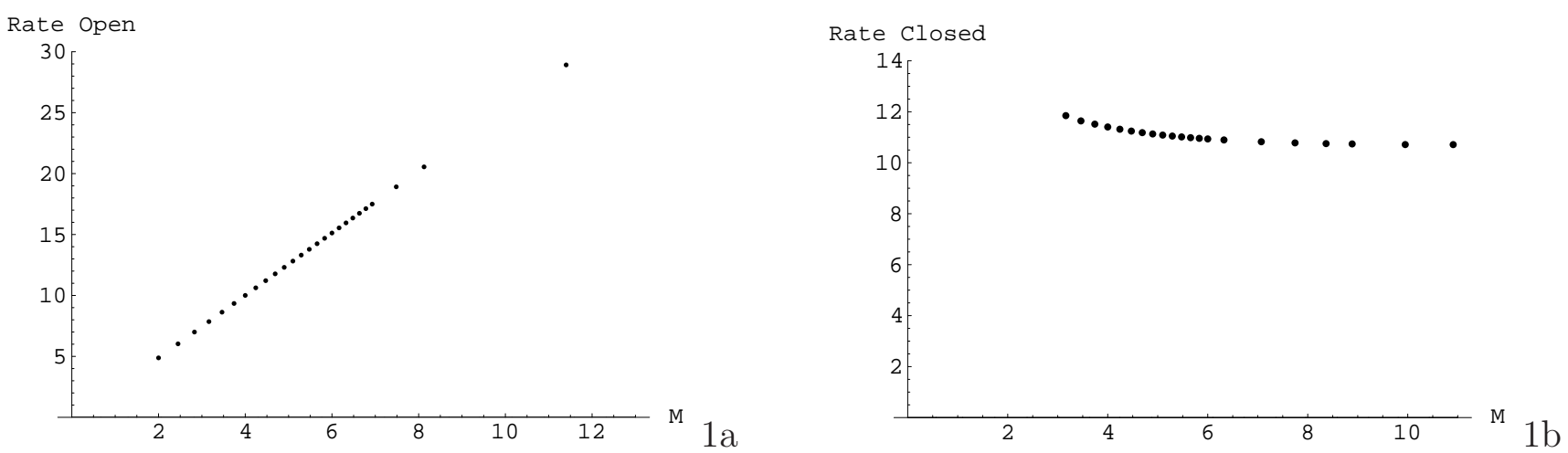

Figure 1: Decay rate versus mass of the open and closed strings with maximum angular momentum. Figure 1a: Open string. Figure 1b: Closed string.

the final string fragments are massive. Therefore, it is of interest to compare the classical prediction with the numerical results restricted to the channels where both decay products are massive.

For the case of the open string the behavior of the rate is quite the same as for the total rate. For the case of the closed string there is something more to comment.

In figs. $2 \mathrm{a}$ and $2 \mathrm{~b}$ we report the decay rate restricted to the massive channels for the closed string with maximal angular momentum with two possible assumptions for the target spacetime. Fig.2a is the case in which every dimension is uncompact. Fig.2b is the case in which six space dimensions are toroidally compactified, the string lying in an uncompact plane (the decay products are taken to have zero winding and KK modes in the compact space).

We see that the decay rate due to massive emission shown in fig.2a is significantly lower than the total decay rate shown in fig.1b. This shows that the decay rate due to massless emission is important (and, at least in this range, decreasing). Now let us compare to the case of $D=4$. We see that flattening towards a constant of the behavior of the massive-channels-lifetime is more evident in Fig.2b (the compactified case) rather than in Fig.2a (with nine uncompact spatial dimensions). It is likely that this is a kinematical effect due to the phase-space being much wider in $D=10$ than in $D=4$ uncompact dimensions. As a result, in $D=10$, getting to the asymptotic regime requires larger masses to overcome the opposite tendency of the phase-space opening. We have also checked that the massless contribution to the decay rate in $D=4$ is larger by a factor $\sim 20$ than the massive contribution, and it is increasing with the mass. This is the expected behavior in $D=4$, as explained in section 2 .

Another interesting case is that of a folded string which is wound $n$ times. In this case, $L=l_{s} \sqrt{N} / n$. The decay rate (1.3) must be multiplied by a factor $\frac{1}{2} 2 n(2 n-1)$, which is the number of possible pairings between $2 n$ folds of the string. This gives

$$
\Gamma_{2 \mathrm{n}-\text { folded }} \cong g_{s}^{2} l_{s}^{-1}(2 n-1)
$$



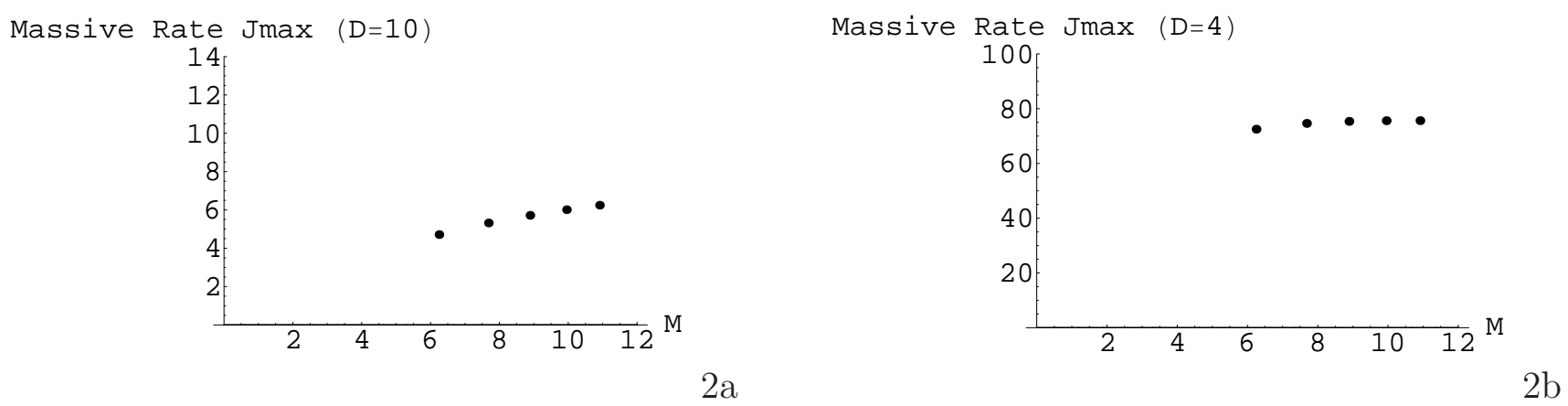

Figure 2: Decay rate versus mass of the closed strings with maximum angular momentum, including massive channels only. Figure 2a: D=10. Figure 2b: $D=4$.

\subsubsection{String which becomes folded at an instant of time}

This is the case of the pulsating and rotating ellipse of section 3, the squashing ellipse. We are interested in the decay rate due to breaking of the string, i.e. into two massive strings. This process is only possible at the instant when the ellipse is completely squashed so that different points of the closed strings get in contact. The time interval where the breaking is possible is of order $l_{s}$ (see section 3). By definition, the decay rate is the number of events at each period of oscillation of the string. This means that the decay rate will be given by

$$
\Gamma_{\text {squash }} \cong \frac{l_{s}}{T} \Gamma_{\text {folded }} \cong \frac{1}{\sqrt{N}} \Gamma_{\text {folded }} \cong g_{s}^{2} \frac{L}{l_{s}^{4} M^{2}} .
$$

In particular, for the specific squashing string of section 3 , one has $L \sim l_{s}^{2} M$, so that

$$
\Gamma_{\text {squash }} \cong g_{s}^{2} \frac{1}{l_{s} \sqrt{N}}
$$

We will find the same law by explicit calculation of the quantum decay in section 3 . This is a non-trivial confirmation of the semiclassical rules given above.

In Fig. 3 we report the results of that computation, in the form of the lifetime (that is, the inverse of the rate) versus mass, restricted to the decay channels where both fragments are massive, following the discussion made for the case of the maximal angular momentum. According to (1.7), one expects a linear behavior, lifetime $\cong$ const. $M$. From fig.3, we see that the quantum calculation reproduces the classical linear behavior even for rather small masses both for $D=10$ and $D=4$ uncompact spacetime dimensions.

\subsubsection{Pulsating circular string}

This is a circular string which shrinks to a point once in a period. The decay rate can be calculated just as in the squashing string case, but now taking into account 

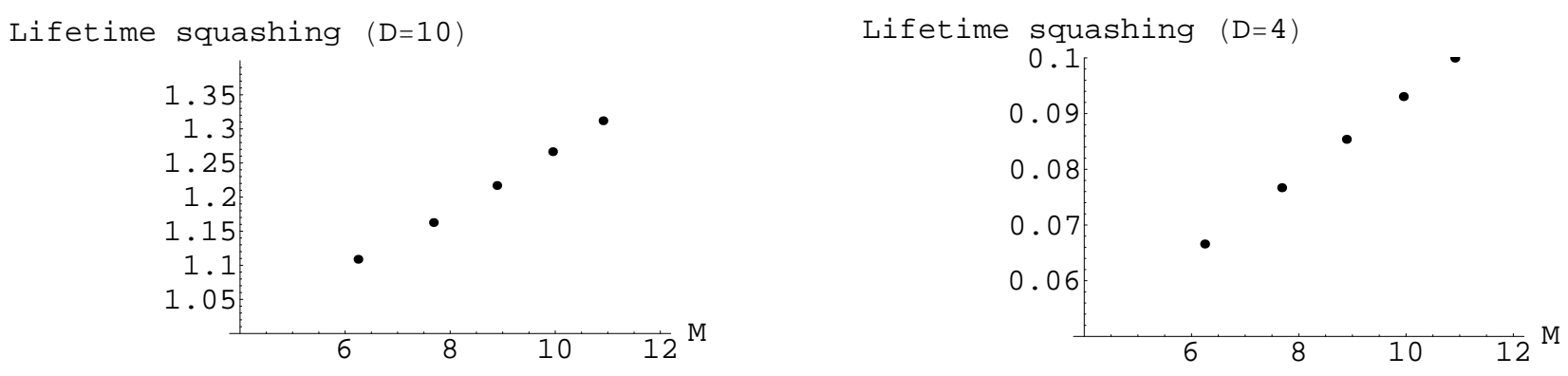

$3 \mathrm{a}$

Figure 3: Lifetime versus mass of the squashing strings, including massive channels only. Figure 3a: $D=10$. Figure 3b: $D=4$.

that the two points where the breaking takes place are arbitrary, since by the time the string is completely shrunk all points are in contact. This means that there is an additional factor of $L / l_{s}$. Thus we get

$$
\Gamma_{\text {pulsating }} \cong g_{s}^{2} \frac{L^{2}}{M^{2} l_{s}^{5}} \cong g_{s}^{2} l_{s}^{-1}
$$

where we have used that $L \cong l_{s}^{2} M$ for the pulsating circular string. Thus the decay rate is the same as in the folded string case with maximum angular momentum. We have verified this remarkable fact by the exact quantum calculation (see section 3).

\subsubsection{Strings with two points in permanent contact}

Let us call this particular closed string configuration a "crossed" closed string. This is similar to the folded string, but now we do not have to multiply by the number of points of the string $L / l_{s}$. Thus we get

$$
\Gamma_{\text {crossed }} \cong g_{s}^{2} \frac{1}{l_{s}^{2} M} \cong g_{s}^{2} \frac{1}{l_{s} \sqrt{N}}
$$

which is similar to the decay rate of the squashing ellipse (1.7).

\subsubsection{Strings with a finite number of contacts in one period}

Let us now consider a string where two points get in contact at an instant of time. The decay rate is obtained by adding a factor $l_{s} / T$ to the previous result (1.9). This factor represents the fraction of time that the strings are in contact, which is the quantum spread $l_{s}$ over the period $T$. Thus

$$
\Gamma \cong g_{s}^{2} \frac{l_{s}}{T^{2}} \cong g_{s}^{2} \frac{1}{l_{s} N} .
$$


For these strings the decay rate due to breaking (1.10) is very small and they are expected to decay primarily by massless radiation.

This case also puts a bound on the decay rate due to splitting of an average closed string state. An average string state can be thought of as a random walk process (see e.g. [4, 5]) and the crossing of the string can occur at most a finite number of times in one period of oscillation. To get the decay rate into massive string states one should multiply the result (1.10) by the probability that any two points meet in one period. Since this number is less than one, this means that, in general, $\bar{\Gamma}_{\text {massive }}<g_{s}^{2} \frac{1}{l_{s} N}$. For these strings, the decay due to massless radiation is largely dominant (see below).

\section{Decay rate due to massless emission}

The quantum massless emission from a closed string contains contributions from four sectors: NS-NS, R-NS, NS-R and R-R. Computing the massless decay rate for a generic quantum string state is in general complicated. In particular, the covariant vertex operator for a general massive string state is not known. We have explicitly computed the decay rate for every channel, including massless emission, in the cases of the string with maximum angular momentum Jmax and for the rotating ring [3, 6], and for the squashing string (section 3).

The rotating ring is a circular string rotating in two orthogonal planes (see the corresponding classical solution in section 4.1). In this case, we found [6] that NS-NS emission (which includes graviton, dilaton and antisymmetric tensor) is dominant, whereas R-NS, NS-R emissions are suppressed by a factor $1 / N$ and R-R emission is suppressed by a factor $1 / N^{2}$, where $N=\alpha^{\prime} M^{2} / 4$ and $M$ is the mass of the decaying string state. Moreover, in this case the NS-NS emission can be accurately described as a radiation process from a classical antenna represented by the classical rotating ring solution.

The classical radiation from a source $T_{\mu \nu}\left(x_{0}, \vec{x}\right)$ in $D$ uncompact spacetime dimensions is given by

$$
\begin{gathered}
\Gamma=g_{s}^{2} \frac{\omega^{D-3}}{M^{2}} \int d^{D-2} \Omega \sum_{\xi, \vec{\xi}}|J|^{2}, \\
J=\int d x_{0} d^{D-1} \vec{x} e^{i \omega X_{0}-i \vec{p} \cdot \vec{X}^{\mu}} \xi^{\mu} \tilde{\xi}^{\nu} T_{\mu \nu}\left(X_{0}, \vec{X}\right) .
\end{gathered}
$$

For a classical string solution, the energy momentum tensor is

$$
T_{\mu \nu}=\int d \sigma d \tau \delta^{(d)}(x-X(\tau, \sigma)) \partial X_{\mu} \bar{\partial} X_{\nu}
$$

so that $|J|^{2}=\left|J_{R}\right|^{2}\left|J_{L}\right|^{2}$ with (gauge $\xi^{0}=\vec{\xi} \cdot \vec{p}=0$ )

$$
J_{R}=\int_{0}^{2 \pi} d \sigma e^{i p_{-} X_{R+}(\sigma)} \vec{\xi} \cdot \partial \vec{X}_{R}^{T}(\sigma)
$$




$$
J_{L}=\int_{0}^{2 \pi} d \sigma e^{i p_{-} X_{L+}(\sigma)} \vec{\xi} \cdot \partial \vec{X}_{L}^{T}(\sigma)
$$

where we have chosen the frame where the momentum of the emitted massless particle is $p^{\mu}=(\omega,-\omega, \overrightarrow{0})$, and the gauge $X_{0}=\alpha^{\prime} M \tau=2 \sqrt{\alpha^{\prime} N} \tau . \quad X_{ \pm}$refer to light-cone coordinates where $p_{+}=0$. The radiated energy is $\omega=\frac{M^{2}-M^{\prime 2}}{2 M}=N_{0} /(2 \sqrt{N})$, where we have set $\alpha^{\prime}=4$ and $N_{0} \equiv N-N^{\prime}$, with $M=\sqrt{N}$ being the mass of the original state and $M^{\prime}=\sqrt{N^{\prime}}$ the mass of the massive state after the emission.

It is important to understand when the classical formula represents a good approximation in order to compute the decay rate due to massless radiation. In other words, which are the string states that radiate like a classical antenna.

The classical formula is expected to hold for $\omega \ll O\left(1 / \sqrt{\alpha^{\prime}}\right)$, i.e. for $N_{0} \ll \sqrt{N}$. In this regime we also expect that NS-R, R-NS and R-R emission is small. If massless NS-NS emission with higher energies is suppressed, then the classical formula can be used to compute the total radiation emission.

In general, since $i p_{-} X_{R, L+}(\sigma) \sim i N_{0} f_{R, L}(\sigma)$ in the exponent in (2.3), (2.4), then $J_{R, L}$ are exponentially suppressed as a function of $N_{0}$, unless there is a saddle point in the integration over $\sigma$ in eqs.(2.3), (2.4), or a "kink" in the function $f_{R, L}(\sigma)$. A saddle point ("cusp") occurs if $\partial_{\sigma} X_{R+}$ and $\partial_{\sigma} X_{L+}$ vanish for some $\sigma$, while a kink occurs when there is discontinuity in the first derivative in the function $f_{R, L}(\sigma)$. This is the case of the cusp and kink string configurations studied in [7, 11.

So let us first assume that there is no cusp or kink. In this case,

$$
J_{R, L}=M h_{R, L}\left(N_{0}, \Omega\right)
$$

where $h_{R, L}\left(N_{0}, \Omega\right)$ are exponentially suppressed for $N_{0} \gg 1$. We have used the fact that $\vec{\xi} \cdot \partial \vec{X}_{R, L}^{T}$ is generically proportional to $M$ as can be seen from the Virasoro constraints. Therefore, the decay rate (2.1) is given by

$$
\Gamma \cong \text { const. } g_{s}^{2} M^{5-D} N_{0}^{D-3} \int d^{D-2} \Omega\left|h_{R}\left(N_{0}, \Omega\right) h_{L}\left(N_{0}, \Omega\right)\right|^{2}
$$

The total rate is obtained by summing over $N_{0}$ (i.e. all possible energies of the massless particle). This sum is convergent and one obtains

$$
\Gamma_{\text {total rad }} \cong \text { const. } g_{s}^{2} M^{5-D}
$$

This law has been verified explicitly in [6] by both the classical and quantum calculation for the rotating ring, which has no cusp or kink. In this case, since decays into massive channels are exponentially suppressed, $\Gamma_{\text {total rad }} \cong \Gamma_{\text {total }}$, so the lifetime of the ring state is $\sim g_{s}^{-2} M^{D-5}$. Note that the time for the state to radiate away all of its energy is much longer by a factor proportional to $N=M^{2}$.

Let us now consider string configurations with cusps. Examples of these are the string with maximum angular momentum $J_{\max }$, the squashing string and the open 
string. In the $J_{\max }$ or the squashing string cases, $\partial \vec{X}_{R, L}^{T}$ vanish for some $\sigma$, which can be taken at $\sigma=0$, behaving as $\partial \vec{X}_{R, L}^{T} \sim \sigma$. Using the Virasoro constraint, $X_{R, L+} \sim \sigma^{3}$. It follows that [7, 11]

$$
J_{R, L}^{\text {cusp }} \sim N^{1 / 2} N_{0}^{-2 / 3} .
$$

Note that the condition of the existence of a cusp depends on the definition of $X_{+}$, which depends on a particular $p^{\mu}=(\omega,-\omega, \overrightarrow{0})$. Therefore the behavior (2.7) holds for a particular angle. Then the classical decay rate (2.1) on this angle becomes

$$
\Gamma\left(N_{0}\right) \sim \text { const. } g_{s}^{2} M^{5-D} N_{0}^{\frac{3 D-17}{3}} .
$$

The sum over $N_{0}$ is convergent only for $D \leq 4$. Therefore, in higher dimensions, in the presence of such cusps, the classical formula cannot be used. In $D=4$, the classical formula for the spectrum is reliable only in the range $N_{0} \ll \sqrt{N}$ (note that the computation of the average value of the emitted energy, proportional to $\left\langle N_{0}\right\rangle$, would be divergent even in $D=4$ at the particular angle for which (2.7) holds). A similar analysis can be carried out for more general string configutations with cusps for which $\partial \vec{X}_{R, L}^{T} \sim \sigma^{\beta}$. This includes the kink case when $\beta=0$. In this case, where $\partial \vec{X}_{R, L}^{T}$ is discontinuous, one gets

$$
J_{R, L}^{\text {kink }} \sim N^{1 / 2} N_{0}^{-1}, \quad \Gamma\left(N_{0}\right) \sim \text { const. } g_{s}^{2} M^{5-D} N_{0}^{D-7} .
$$

String configurations with kinks appear naturally as a result of the splitting of a string [10. The kink or cusp feature is preserved during the classical evolution of a string. However, from the above formulas we see that kink and cusp string configurations have a radiation spectrum which does not decay exponentially. As a result, such strings radiate more, providing a dissipation mechanism which should smooth out the kinks and cusps quantum mechanically.

Whenever the sum over $N_{0}$ is convergent for every angle one obtains again, either in the cusp or kink case, $\Gamma_{\text {total rad }} \cong$ const. $g_{s}^{2} M^{5-D}$. By comparing with the exact numerical results of the full quantum computation, we have verified that this is indeed the behavior in $D=4$ for Jmax and the squashing string, while this is not the behavior of $\Gamma_{\text {total rad }}$ in $D=10$, as expected.

Finally, another case where the massless radiation emission can be computed explicitly is that of an average string state. In this case, one finds [9, 6]

$$
\bar{\Gamma}_{\text {total rad }} \cong g_{s}^{2} M
$$

This formula is the same even if some dimensions are compact. It was also derived in [1] from a study of the mass shift.

In the previous section we have made an estimate for the decay rate due to breaking for an average string state $\bar{\Gamma}_{\text {massive }}<g_{s}^{2} \frac{1}{l_{s} N}$. This is much smaller than $\bar{\Gamma}_{\text {total rad }}$ so for an average string state the lifetime will be determined by the massless radiation channel, i.e. given by $\mathcal{T}=\left(g_{s}^{2} M\right)^{-1}$. 


\section{The squashing ellipse}

The classical string solution is given by

$$
\begin{aligned}
& x_{1}=2 L \cos \theta \cos \tau \cos \sigma, \quad x_{2}=2 L \sin \tau \cos \sigma, \\
& x_{3}=2 L \sin \theta \sin \tau \sin \sigma, \quad x_{0}=2 L \tau,
\end{aligned}
$$

where $\theta$ is a parameter and $\sigma \in[0,2 \pi)$. For $\theta$ generic, it describes an ellipse that rotates around one of its axes and simultaneously performs pulsations, with one of its radii (the one on the axes of rotation) becoming zero at $\tau=n \pi, n=$ integer.

This string interpolates between the folded string with $\theta=0$, so that

$$
\begin{aligned}
& x_{1}=2 L \cos \tau \cos \sigma, \quad x_{2}=2 L \sin \tau \cos \sigma, \\
& x_{3}=0, \quad x_{0}=2 L \tau,
\end{aligned}
$$

and the pulsating circular string with $\theta=\pi / 2$,

$$
\begin{aligned}
& x_{1}=0, \quad x_{2}=2 L \sin \tau \cos \sigma, \\
& x_{3}=2 L \sin \tau \sin \sigma, \quad x_{0}=2 L \tau .
\end{aligned}
$$

At every period of oscillation, there are two times where the string (3.1) becomes folded and it can break. Quantum mechanically, the breaking process is important during the time that the smaller radius of the ellipse has size $<l_{s}$. As it is clear from the above solution, this occurs during a time $\Delta x_{0} \cong l_{s}$ at each period. So the fraction of time where the string can break is $l_{s} / L$. This was used in section 1 to estimate the lifetime by semiclassical arguments.

\subsection{Classical breaking}

Let us now work out the masses of the outgoing string solutions after the splitting. The ellipse becomes folded at $\tau=0$, where

$$
x_{1}(0, \sigma)=2 L \cos \theta \cos \sigma, \quad x_{2}(0, \sigma)=x_{3}(0, \sigma)=0 .
$$

We assume that the string breaks at $\sigma=a \pi$ and $\sigma=2 \pi-a \pi, 0<a<1$, corresponding to the same spacetime point, because the string is folded. Following [10, the outgoing strings I and II and their masses can be determined by requiring continuity of $x_{1}, x_{2}, x_{3}$ and $\dot{x}_{1}, \dot{x}_{2}, \dot{x}_{3}$. For the first derivatives, we have

$$
\dot{x}_{1}(0, \sigma)=0, \quad \dot{x}_{2}(0, \sigma)=2 L \cos \sigma, \quad \dot{x}_{3}(0, \sigma)=2 L \sin \theta \sin \sigma .
$$

Now we compute the linear momenta of the outgoing strings I and II. Since they are conserved, they can be computed at $\tau=0$. The components of the momenta are

$$
p_{\mathrm{I}}^{x_{2}}=\frac{2}{2 \pi \alpha^{\prime}} \int_{0}^{\pi a} d \sigma \dot{x}_{2}=\frac{4 L}{2 \pi \alpha^{\prime}} \int_{0}^{\pi a} d \sigma \cos \sigma=\frac{2 L}{\pi \alpha^{\prime}} \sin \pi a
$$




$$
\begin{gathered}
p_{\mathrm{I}}^{x_{1}}=0, \quad p_{\mathrm{I}}^{x_{3}}=\frac{1}{2 \pi \alpha^{\prime}}\left(\int_{0}^{\pi a}+\int_{2 \pi-\pi a}^{2 \pi}\right) d \sigma \dot{x}_{3}=0 \\
E_{\mathrm{I}}=\frac{2}{2 \pi \alpha^{\prime}} \int_{0}^{\pi a} d \sigma \dot{x}_{0}=\frac{2 L a}{\alpha^{\prime}}
\end{gathered}
$$

Hence

$$
M_{\mathrm{I}}^{2}(a)=E_{\mathrm{I}}^{2}-\left(p_{\mathrm{I}}^{x_{2}}\right)^{2}=\frac{4 L^{2}}{\alpha^{\prime 2}}\left(a^{2}-\frac{\sin ^{2} \pi a}{\pi^{2}}\right)
$$

Similarly

$$
M_{\mathrm{II}}^{2}(a)=\frac{4 L^{2}}{\alpha^{\prime 2}}\left((1-a)^{2}-\frac{\sin ^{2} \pi a}{\pi^{2}}\right) .
$$

Thus the masses of the two outgoing strings are the same for any $\theta$. In the case $\theta=0$, we recover the results of the folded string. The opposite situation is when $\theta=\pi / 2$, and we recover the results of the pulsating circular string [12].

In fig. 4 we show the strings I and II at a generic time after the splitting, for $\theta=\pi / 4$ (representing a generic squashing ellipse). The strings I and II rotate and pulsate, and thet have two kinks which travel along the string. Remarkably, at periodic times, they become folded.

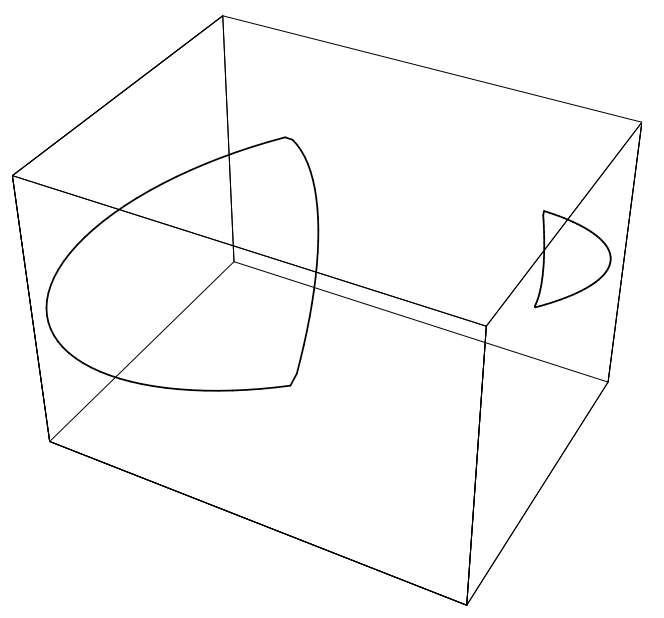

Figure 4: Fragments after the splitting of the squashing string.

\subsection{Quantum decay for the squashing ellipse}

The squashing string solution can be written in terms of Right and Left components as follows

$$
\begin{aligned}
x_{1 L}=L \cos \theta \cos \left(\sigma_{+}\right), & x_{1 R}=L \cos \theta \cos \left(\sigma_{-}\right), \\
x_{2 L}=L \sin \left(\sigma_{+}\right), & x_{2 R}=L \sin \left(\sigma_{-}\right), \\
x_{3 L}=-L \sin \theta \cos \left(\sigma_{+}\right), & x_{3 R}=L \sin \theta \cos \left(\sigma_{-}\right),
\end{aligned}
$$


where $\sigma_{ \pm}=\tau \pm \sigma$. It is convenient to define

$$
\begin{aligned}
& u_{R}=\frac{1}{\sqrt{2}}\left(x_{2 R}+i\left(\cos \theta x_{1 R}+\sin \theta x_{3 R}\right)\right), \\
& v_{L}=\frac{1}{\sqrt{2}}\left(x_{2 L}+i\left(\cos \theta x_{1 L}-\sin \theta x_{3 L}\right)\right) .
\end{aligned}
$$

In type II superstring theory, one can derive the following formula for $\Delta M^{2}$ at one loop [13, 3]:

$$
\Delta M^{2}=c g_{s}^{2} \int \frac{d^{2} \tau}{\tau_{2}^{3}} \int d^{2} z \sum_{s}\left\langle\hat{V}^{\dagger}(z) \hat{V}(0)\right\rangle_{s}
$$

where $\hat{V}$ is the (super) vertex operator of the string state and we have indicated the sum over the spin structures $s$. The decay rate is then obtained as $\Gamma=\operatorname{Im} \Delta M^{2} /(2 M)$. [It is also possible -and we have done it in several cases- to compute the decay rate for each of the channels NS-NS, N-R, R-NS, R-R, that is, for each spin structure separately.]

After evaluating $\left\langle e^{-i p \cdot X(z)} e^{i p \cdot X(0)}\right\rangle$, the world-sheet fermion correlators and summing over the spin structures, the formula (3.13) reduces to

$$
\Delta M^{2}=c g_{s}^{2} \int \frac{d^{2} \tau}{\tau_{2}^{3}} \int d^{2} z e^{-4 N \frac{\pi(\operatorname{Im} z)^{2}}{\tau_{2}}}\left|\frac{\theta_{1}(z \mid \tau)}{\theta_{1}^{\prime}(0 \mid \tau)}\right|^{4 N}\left\langle W^{\dagger}(z) W(0)\right\rangle,
$$

where, in terms of $u_{R}, v_{L}$, the effective bosonic vertex operator $W$ corresponding to the quantum state that describes the squashing ellipse is given by

$$
\begin{gathered}
W=\left(\frac{2}{\alpha^{\prime}}\right)^{N-1} N V_{R} V_{L} \\
V_{R}=\left(\partial u_{R}\right)^{n}, \quad V_{L}=\left(\bar{\partial} v_{L}\right)^{n}, \quad n=N-1 .
\end{gathered}
$$

Now let us calculate the correlator

$$
\left\langle W^{\dagger}(z) W(0)\right\rangle=\left\langle\left(\bar{\partial} v_{L}^{*}(\bar{z})\right)^{n}\left(\partial u_{R}^{*}(z)\right)^{n}\left(\partial u_{R}(0)\right)^{n}\left(\bar{\partial} v_{L}(0)\right)^{n}\right\rangle .
$$

The non-zero correlators are:

$$
\begin{aligned}
\left\langle\partial u_{R}^{*}(z) \partial u_{R}(0)\right\rangle & =\frac{\alpha^{\prime}}{2}\left(\partial^{2} \log \theta_{1}(z)+\frac{\pi}{\tau_{2}}\right) \\
\left\langle\bar{\partial} v_{L}^{*}(\bar{z}) \bar{\partial} v_{L}(0)\right\rangle & =\frac{\alpha^{\prime}}{2}\left(\bar{\partial}^{2} \log \theta_{1}(\bar{z})+\frac{\pi}{\tau_{2}}\right) \\
\left\langle\partial u_{R}^{*}(z) \bar{\partial} v_{L}(0)\right\rangle & =\left\langle\bar{\partial} v_{L}^{*}(z) \partial u_{R}(0)\right\rangle=\frac{\alpha^{\prime}}{2} \frac{\pi}{\tau_{2}} \cos ^{2} \theta \\
\left\langle\bar{\partial} v_{L}(0) \partial u_{R}(0)\right\rangle & =\left\langle\partial u_{R}^{*}(z) \bar{\partial} v_{L}^{*}(\bar{z})\right\rangle=\frac{\alpha^{\prime}}{2} \frac{\pi}{\tau_{2}} \sin ^{2} \theta .
\end{aligned}
$$


In (3.19) we have regularized in a way consistent with (3.18). We obtain

$$
\begin{aligned}
\left\langle W^{\dagger}(z) W(0)\right\rangle & =\left(\frac{2}{\alpha^{\prime}}\right)^{2 N-2} \sum_{m_{1}, m_{2}} \frac{N^{2} n !^{4}}{m_{1} !^{2} m_{2} !^{2}\left(n-m_{1}-m_{2}\right) !^{2}}\left\langle\partial u_{R}^{*}(z) \bar{\partial} v_{L}^{*}(\bar{z})\right\rangle^{m_{1}}\left\langle\bar{\partial} v_{L}(0) \partial u_{R}(0)\right\rangle^{m_{1}} \\
& \times\left\langle\partial u_{R}^{*}(z) \bar{\partial} v_{L}(0)\right\rangle^{m_{2}}\left\langle\bar{\partial} v_{L}^{*}(z) \partial u_{R}(0)\right\rangle^{m_{2}}\left(\left\langle\partial u_{R}^{*}(z) \partial u_{R}\right\rangle\left\langle\bar{\partial} v_{L}^{*}(\bar{z}) \bar{\partial} v_{L}\right\rangle\right)^{n-m_{1}-m_{2}}
\end{aligned}
$$

Thus

$$
\left\langle W^{\dagger}(z) W(0)\right\rangle=\sum_{l_{L}, l_{R}=0}^{N-1} Q\left(N, \theta, l_{L}, l_{R}\right)\left(\frac{\pi}{\tau_{2}}\right)^{2 N-2-l_{R}-l_{L}}\left(\partial^{2} \log \theta_{1}(z)\right)^{l_{R}}\left(\bar{\partial}^{2} \log \theta_{1}(\bar{z})\right)^{l_{L}},
$$

with

$$
Q\left(N, \theta, l_{L}, l_{R}\right)=\sum_{m_{1}, m_{2}} \frac{N !^{2}(\sin \theta)^{4 m_{1}}(\cos \theta)^{4 m_{2}}}{m_{1} !^{2} m_{2} !^{2} l_{R} !_{L} !\left(N-1-m_{1}-m_{2}-l_{R}\right) !\left(N-1-m_{1}-m_{2}-l_{L}\right) !}
$$

Note that $\theta=0$ and $\theta=\frac{\pi}{2}$ give identical expressions. This shows the remarkable fact that the quantum decay rate of the pulsating circular string and that of the folded string with maximum angular momentum are identical, confirming the semiclassical estimate of section 1.

Next, one needs to integrate over $\tau$ and $z$. This is done in two steps 13, 3. One expands the holomorphic and antiholomorphic parts of $\left|\theta_{1}(z \mid \tau) / \theta_{1}^{\prime}(0 \mid \tau)\right|^{4 N}\left\langle W^{\dagger}(z) W(0)\right\rangle$ in powers of $e^{i 2 \pi \tau}$ and $e^{i 2 \pi z}$. The integrals over real parts of $\tau$ and $z$ set the same power for each term in the holomorphic and antiholomorphic parts. The integral over the imaginary part of $z$ is a gaussian, and the integral over the imaginary part of $\tau$ is carried out by using

$$
\operatorname{Im} \int d \tau_{2} \tau_{2}^{-\alpha} e^{\tau_{2} A}=\frac{\pi A^{\alpha-1}}{\Gamma(\alpha)} .
$$

We refer to [13, 3] for more details. The results of the numerical evaluation of the final expressions are summarized in Table 1, which shows the contribution of the massive channels (we have taken $\theta=\pi / 4$, as a representative of a typical squashing string). Here we are only interested in massive channels, since we wish to compare with the semiclassical estimate of section 1.

\begin{tabular}{l|l|l|}
$\mathrm{N}$ & $\Gamma_{\text {Jmax }}$ & $\Gamma_{\text {squash }}$ \\
\hline 39 & 4.713 & 0.903 \\
59 & 5.321 & 0.860 \\
79 & 5.718 & 0.822 \\
99 & 6.013 & 0.789 \\
119 & 6.250 & 0.762
\end{tabular}

Table 1: Sum over massive channels $(\theta=\pi / 4)$. 
Figure 5 shows the plot of the ratio between $\Gamma_{\text {Jmax }}$ and $\Gamma_{\text {squash }}$ as a function of the mass $M=\sqrt{N}$. According to the semiclassical estimate (1.4), (1.7) of section 1, $\Gamma_{\text {Jmax }} / \Gamma_{\text {squash }}$ should depend linearly on $\sqrt{N}$. We see that this is in perfect agreement with figure 5. Figures $3 \mathrm{a}$ and $3 \mathrm{~b}$, showing that the lifetime of the squashing string $\Gamma_{\text {squash }}^{-1}$ is linear with $\sqrt{N}$, add more evidence to the law $\Gamma_{\text {squash }} \sim N^{-1 / 2}$. Thus the explicit quantum calculation confirms the semiclassical argument of section 1.

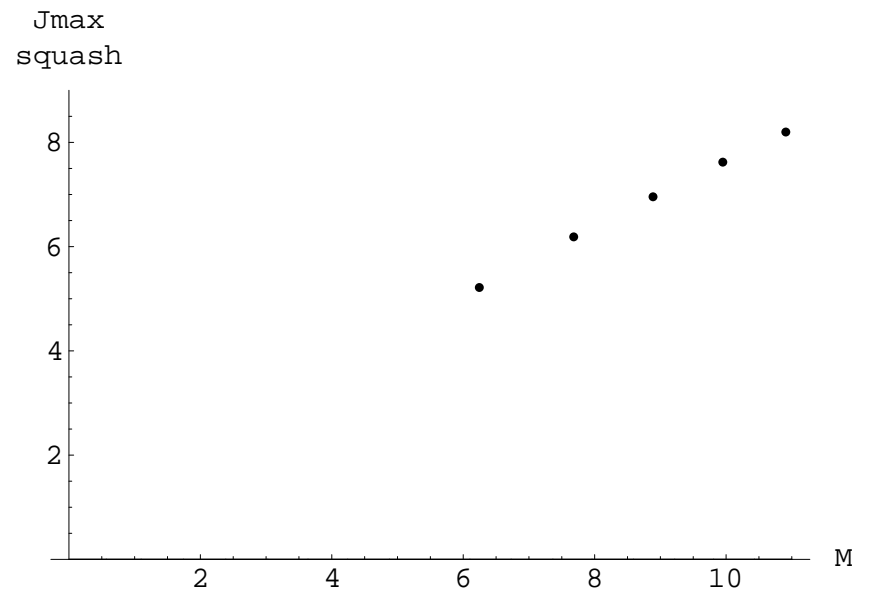

Figure 5: Ratio of decay rates $\Gamma_{\mathrm{Jmax}} / \Gamma_{\mathrm{squash}}$ as a function of $M=\sqrt{N}$.

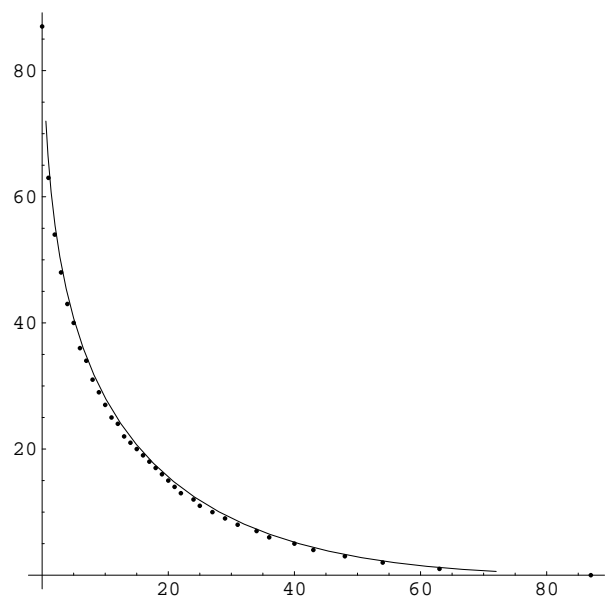

Figure 6: Maxima of $\Gamma\left(M_{\mathrm{I}}, M_{\mathrm{II}}\right)$ and curve of classical splitting (3.9), (3.10) for the squashing string.

The quantum decay rate (3.14 can be written as a sum over decay channels $\Gamma\left(M_{\mathrm{I}}, M_{\mathrm{II}}\right)$ in which the string decays into two fragments of masses $M_{\mathrm{I}}, M_{\mathrm{II}}$. We find that, as in 13, $\Gamma\left(M_{\mathrm{I}}, M_{\mathrm{II}}\right)$ is exponentially small $O\left(\exp \left(-c M^{2}\right)\right)$ except on a curve in the plane $M_{\mathrm{I}}, M_{\mathrm{II}}$, where it has a maximum. This curve precisely corresponds to the curve (3.9), (3.10) determined by the classical splitting, showing that for large $M$ the classical splitting is the dominant process. Figure 6 shows the comparison of the maxima of $\Gamma\left(M_{\mathrm{I}}, M_{\mathrm{II}}\right)$ with the classical curve (3.9), (3.10) for the case of the squashing string (the same comparison for the case of Jmax has been done in [10]). 


\section{General class of rotating and pulsating closed strings}

There is a simple class of solutions of strings rotating in two planes which contain the cases of the rotating ring, the folded string with maximum angular momentum, and the squashing ellipses. This class of solutions includes as particular cases various solutions which have appeared in the literature (see e.g. recently [14]). In general, the solution represents a string of elliptical shape which rotates and pulsates at the same time. The solution is given by

$$
\begin{aligned}
Z_{1} & =\frac{X_{1}+i X_{2}}{\sqrt{2}}=L\left(\cos \alpha \cos \beta e^{i \sigma_{-}}+\sin \alpha \sin \beta e^{-i \sigma_{-}+i \gamma}\right. \\
& \left.+\sin \alpha \cos \beta e^{i \sigma_{+}}-\cos \alpha \sin \beta e^{-i \sigma_{+}-i \gamma}\right), \\
Z_{2} & =\frac{X_{3}+i X_{4}}{\sqrt{2}}=L\left(\sin \alpha \cos \beta e^{i \sigma_{-}}-\cos \alpha \sin \beta e^{-i \sigma_{-}+i \gamma}\right. \\
& \left.+\cos \alpha \cos \beta e^{i \sigma_{+}}+\sin \alpha \sin \beta e^{-i \sigma_{+}-i \gamma}\right), \\
X_{0} & =2 L \tau=L\left(\sigma_{+}+\sigma_{-}\right), \quad \sigma_{ \pm}=\tau \pm \sigma, \quad \sigma \in[0,2 \pi) .
\end{aligned}
$$

The solution is thus characterized by four parameters $(L, \alpha, \beta, \gamma)$. The mass of the classical solution (4.1) is given by

$$
M=\frac{2 L}{\alpha^{\prime}}
$$

There are six conserved angular momentum components,

$$
J_{i j}=\frac{1}{2 \pi \alpha^{\prime}} \int_{0}^{2 \pi}\left(X_{i} \dot{X}_{j}-X_{j} \dot{X}_{i}\right) .
$$

We obtain

$$
\begin{gathered}
J_{12}=J_{34}=\frac{L^{2}}{\alpha^{\prime}} \cos 2 \beta \\
J_{24}=J_{31}=\frac{L^{2}}{\alpha^{\prime}} \sin 2 \beta \sin \gamma \\
J_{14}=J_{23}=\frac{L^{2}}{\alpha^{\prime}} \sin 2 \alpha
\end{gathered}
$$

At any given instant of time, the closed string is an ellipse. By performing an $S O(4)$ transformation one can go to an instantaneous frame where the solution takes the form

$$
\tilde{Z}_{1}=L_{1}(\tau) \cos \left(\sigma-\sigma_{0}\right)+i L_{2}(\tau) \sin \left(\sigma-\sigma_{0}\right), \quad \tilde{Z}_{2}=0
$$


The functions $L_{1}(\tau), L_{2}(\tau)$ give the two radii of the ellipse at any time. To determine them, we use

$$
Z_{1} Z_{1}^{*}+Z_{2} Z_{2}^{*}=\tilde{Z}_{1} \tilde{Z}_{1}{ }^{*}+\tilde{Z}_{2} \tilde{Z}_{2}{ }^{*}=L_{1}^{2}(\tau) \cos ^{2}\left(\sigma-\sigma_{0}\right)+L_{2}^{2}(\tau) \sin ^{2}\left(\sigma-\sigma_{0}\right)
$$

and inserting (4.1) we find

$$
L_{1,2}^{2}(\tau)=2 L^{2}\left(1-\cos (\gamma) \cos (2 \alpha) \sin (2 \beta) \cos (2 \tau) \pm \sin (2 \alpha) \sqrt{1-\cos ^{2}(\gamma) \sin ^{2}(2 \beta)}\right)
$$

The ellipse contracts to a minimum size, whose smaller radius is

$$
L_{2 \min }^{2}=2 L^{2}\left(1-\cos (\gamma) \cos (2 \alpha) \sin (2 \beta)-\sin (2 \alpha) \sqrt{1-\cos ^{2}(\gamma) \sin ^{2}(2 \beta)}\right)
$$

where we assumed $\sin (2 \alpha)>0$ and $\cos (\gamma) \cos (2 \alpha) \sin (2 \beta)>0$.

If $L_{2 \min } \neq 0$, the closed string cannot break, because there are never two points of the closed string that get in contact. In the special case that $L_{2 \min }$ is equal to zero, the ellipse contracts to become a folded string, and in this case it can break. This is the squashing ellipse discussed in section 3 . If, in addition, $L_{1 \text { min }}=0$, then the string contracts to a point. This latter solution is the pulsating circular string.

In the case $\gamma=0$ there are some simplifications. One gets

$$
L_{1,2}^{2}(\tau)=2 L^{2}(1-\cos (2 \alpha) \sin (2 \beta) \cos (2 \tau) \pm \sin (2 \alpha) \cos (2 \beta)) .
$$

We may assume without loss of generality that $\sin (2 \alpha) \cos (2 \beta) \geq 0$, since the opposite sign gives an equivalent solution. Then $L_{2}(\tau) \leq L_{1}(\tau)$. The $\gamma=0$ ellipse contracts to a minimum size where the smaller radius is given by

$$
L_{2 \min }^{2}= \begin{cases}2 L^{2}(1-\sin 2(\beta+\alpha)), & \cos 2 \alpha \sin 2 \beta>0 \\ 2 L^{2}(1+\sin 2(\beta+\alpha)), & \cos 2 \alpha \sin 2 \beta<0\end{cases}
$$

In what follows we discuss different special cases separately.

\subsection{Pulsating and rotating rings}

The ellipse becomes a circle, with $L_{1}(\tau)=L_{2}(\tau)$ for all $\tau$, in the cases: a) $\sin 2 \alpha=0$ or b) $\gamma=0$ and $\cos (2 \beta)=0$. In this case $L_{1}(\tau)=L_{2}(\tau)$.

These solutions describe a circular string which, combined with the rotational motion, periodically contracts to a minimum radius (which, generically, is different from zero) and then expands to a maximum radius.

Consider $\alpha=0$. Then, by introducing new complex coordinates

$$
\tilde{Z}_{1}=\frac{Z_{1}+Z_{2}^{*}}{\sqrt{2}}, \quad \tilde{Z}_{2}=\frac{Z_{1}-Z_{2}^{*}}{\sqrt{2}},
$$


we obtain

$$
\begin{array}{ll}
\tilde{Z}_{1}=L_{1} e^{-i \sigma} \cos (\tau), & L_{1}=\sqrt{2} L\left(\cos \beta-e^{-i \gamma} \sin \beta\right), \\
\tilde{Z}_{2}=i L_{2} e^{-i \sigma} \sin (\tau), & L_{2}=\sqrt{2} L\left(\cos \beta+e^{-i \gamma} \sin \beta\right) .
\end{array}
$$

It contains the rotating ring and the pulsating circular string as the special cases $\beta=0$ and $\beta=\frac{\pi}{4}, \gamma=0$, respectively.

\subsection{Rotating rigid ellipses}

The ellipses do not pulsate when

$$
\cos (\gamma) \cos (2 \alpha) \sin (2 \beta)=0
$$

which is satisfied for $\gamma=\frac{\pi}{2}$ or $\beta=0$ or $\alpha=\frac{\pi}{4}$. Then $L_{1}, L_{2}$ are constants given by

$$
L_{1,2}^{2}=2 L^{2}\left(1 \pm \sin (2 \alpha) \sqrt{1-\cos ^{2}(\gamma) \sin ^{2}(2 \beta)}\right) .
$$

These rotating rigid ellipses are equivalent to the solutions of [3]. In this work, the quantum decay rate for the corresponding quantum states was computed by explicit evaluation of $\operatorname{Im}\left(\Delta M^{2}\right)$ at one loop.

\subsection{Squashing ellipses}

The solution represents an ellipse that contracts to a folded closed string, i.e. with $L_{2 \min }^{2}=0$, when

$$
\cos (\gamma) \cos (2 \alpha) \sin (2 \beta)+\sin (2 \alpha) \sqrt{1-\cos ^{2}(\gamma) \sin ^{2}(2 \beta)}=1
$$

The solution of this equation is

$$
\cos (\gamma)=\frac{\cos (2 \alpha)}{\sin (2 \beta)}
$$

The solution discussed in section 3 corresponds to $\gamma=0$. Then the condition (4.16) simplifies to

$$
\sin 2(\beta+\alpha)=1, \quad \text { or } \quad \beta+\alpha=\frac{\pi}{4} .
$$

Introducing new complex coordinates

$$
z_{1}=x_{1}+i x_{2}=\frac{Z_{1}+Z_{2}}{\sqrt{2}}, \quad z_{2}=x_{3}+i x_{4}=\frac{Z_{1}-Z_{2}}{\sqrt{2}},
$$

and using $\beta=\frac{\pi}{4}-\alpha$, we find

$$
\begin{array}{ll}
x_{1}=2 L \sin 2 \alpha \cos \tau \cos \sigma, & x_{2}=2 L \sin \tau \cos \sigma, \\
x_{3}=2 L \cos 2 \alpha \sin \tau \sin \sigma, & x_{4} \equiv 0
\end{array}
$$

which is equivalent to (3.1) with $\theta=2 \alpha+\pi / 2$. Thus in this special case the string (4.1) moves in three spatial dimensions $\left(x_{1}, x_{2}, x_{3}\right)$. 


\section{Acknowledgments}

We would like to thank D. Chialva for discussions on related topics. This work is supported in part by the European EC-RTN network MRTN-CT-2004-005104. J.R. also acknowledges support by MCYT FPA 2004-04582-C02-01 and CIRIT GC 2005SGR-00564.

\section{References}

[1] J. Dai and J. Polchinski, "The Decay Of Macroscopic Fundamental Strings," Phys. Lett. B 220, 387 (1989).

[2] H. Okada and A. Tsuchiya, "The Decay Rate Of The Massive Modes In Type I Superstring," Phys. Lett. B 232, 91 (1989).

[3] D. Chialva, R. Iengo and J. G. Russo, "Decay of long-lived massive closed superstring states: Exact results," JHEP 0312, 014 (2003) arXiv:hep-th/0310283.

[4] D. Mitchell and N. Turok, "Statistical Properties Of Cosmic Strings," Nucl. Phys. B 294, 1138 (1987).

[5] J. L. Manes, "Portrait of the string as a random walk," JHEP 0503, 070 (2005) arXiv:hep-th/0412104.

[6] D. Chialva, R. Iengo and J. G. Russo, "Search for the most stable massive state in superstring theory," JHEP 0501, 001 (2005) arXiv:hep-th/0410152.

[7] A. Vilenkin and E.P.S. Shellard, "Cosmic strings and other topological defects", (Cambridge University press 1994).

[8] T. Damour and A. Vilenkin, "Gravitational wave bursts from cusps and kinks on cosmic strings," Phys. Rev. D 64, 064008 (2001) arXiv:gr-qc/0104026.

[9] D. Amati and J. G. Russo, "Fundamental strings as black bodies," Phys. Lett. B 454, 207 (1999) arXiv:hep-th/9901092.

[10] R. Iengo and J. G. Russo, "Semiclassical decay of strings with maximum angular momentum," JHEP 0303, 030 (2003) arXiv:hep-th/0301109.

[11] T. Damour and G. Veneziano, "Self-gravitating fundamental strings and black holes," Nucl. Phys. B 568, 93 (2000) arXiv:hep-th/9907030.

[12] H. J. de Vega, J. Ramirez Mittelbrunn, M. Ramon Medrano and N. Sanchez, "Classical splitting of fundamental strings," Phys. Rev. D 52, 4609 (1995).

[13] R. Iengo and J. G. Russo, "The decay of massive closed superstrings with maximum angular momentum," JHEP 0211, 045 (2002) arXiv:hep-th/0210245. 
[14] G. Niz and N. Turok, "Classical propagation of strings across a big crunch / big bang singularity," arXiv:hep-th/0601007. 